\title{
The Vacuum-Phonon (Sonon) Reinterpretation of QED
}

\author{
Terry B. Bollinger (1) 1 \\ ${ }^{1}$ Apabistia Research, Ashburn, VA, USA 20147
}

(Incepted 26 September 2021; Published 26 September 2021; Tweaked 2021-09-27.13:02)*

\begin{abstract}
This paper provides a reference copy of one particular and highly informal comment in a multiweek Academia.edu discussion of the paper Randomness in Relational Quantum Mechanics by Gary Gordon. The other main participants in this particular thread of the discussion were Doug Marman, Conrad Dale Johnson, Ruth Kastner, and the author. In this comment, the author argues that the only self-consistent approach to reconciling Feynman path integrals with Maxwell's experimentally well-proven theory of electromagnetic wave pressure is introducing a new spin-0 particle, the vacuum or space phonon (sonon), that conveys linear momentum. The path histories of QED become the always-expanding structure of the sonon field, which, like a bubble, becomes increasingly unstable as it expands. The collection of all sonon fields around well-defined bundles of conserved quantum properties creates xyz space by defining the complete set of relational information for those entities. Spacetime in the sonon model is granular, multiscale, and entirely mass-energy dependent. Implications of the sonon model are discussed, including the need for a drastic update to general relativity to take the multi-scale granularity of spacetime directly into account, rather than explaining it obliquely via models such as dark matter, dark energy, or MOND.
\end{abstract}

DOI: https://doi.org/10.48034/20210926

\section{INTRODUCTION}

2021-09-26.11:15 Sun

Gary, Doug, [and Dr. Kastner]

Gary, with regards to the photon absorption issue:

You are correct. Doug, Dr. Kastner, and Earlier Terry are all wrong on this one: Electrons absorb photons, not mirrors.

The simplest reason to see why is the observation that mirrors have no net charge, and thus cannot "see" electrons in the QED sense. They also have insanely wrong masses for resonating with photons.

The only mirror particles with any chance of absorbing light-spectrum photons in the QED sense of dismantling them into raw quantum numbers are electrons. Only electrons have the right combination of electric charge and sufficiently light mass to resonate with and absorb lightspectrum photons. The proof that electrons in material

Published by Apabistia Press under the Creative Commons Attribution 4.0 International License. You are free to redistribute this article as you choose, provided only that you preserve the author attribution, article title, journal citation, and DOI.

${ }^{*}$ This document uses Kenobi versioning. The online name always points to the most recent document version, while obl, $o b 2, \ldots$ suffixes indicate obsolete releases of the document.
This is about: Schrödinger equation, Compton scattering, Solar sails, Quantum teleportation, Quantum theory, Quantum cosmology, General relativity [NEW] Vacuum phonon, Space phonon, Sonon

objects absorb photons is called color.

Protons in mirrors of course also have an electric charge and so can "see" light-spectrum photons. However, the protons are far too heavy to resonate well with photons in the light range, and in every element except hydrogen, they are also extremely well shielded from photons by the inner shell electrons. Thus it's a pretty safe approximation to say the protons in mirrors don't absorb light-spectrum photons.

But here is the problem: Reflection is not, and can never be, the same thing as absorption.

The problem is that part about "dismantling quantum numbers." When an electron in a colored object absorbs a photon, it also absorbs the spin of the photon, causing the electron (at least in some cases) to flip from spin-up to spin-down as the whole spin is absorbed. The entire dismantling operation is extremely localized in xyz space, down to the atom in which the flipped electron resides.

Reflection does not dismantle photons in this same sense. When a mirror reflects a photon, no specific, localized electron ever dismantles and picks up its spin. On a much smaller size scale, the same statement is true for coherent Compton scattering in which the photon "reflects" off of the atom without any of the electrons in the atom picking up its spin. If such an electron does pick up that spin, you get either ionization or a Rydberg atom. 
Notice also that in coherent Compton scattering the "mirror" once again has no net charge, and thus is incapable, as a whole, of "absorbing" the photon. Coherent Compton scattering is, in short, just a very small-scale version of mirror reflection.

The dismantling issue is even more vivid in the case of neutron reflection. A neutron is a complicated beast, and it can only truly be absorbed if a single atomic nucleus exerts the strong force to bind it.

That is not what happens in neutron reflection. The neutron must choose between absorption and reflection. It is either firmly and classically bound by the strong force to one atomic nucleus, or it reflects off of the entire liquid mercury surface without engaging in the dismantling or adhering process.

Reflection is much more akin to the situation in the twoslit experiment. It's not what does happen that counts, but what does not happen. The electron in the two-slit experiment does not collide with the mask, but instead "could" be going through either slit. When no history is recorded, quantum interference and path integrals kick in instead.

Thus a photon hitting a mirror "sees" the mirror because it could be absorbed by any of an enormous number of electrons in the mirror, but is not actually absorbed by any of them! It doesn't matter much at all whether the potential absorption electrons are atomically bound (diffraction mirrors) or delocalized (Fermi surface band electrons in metals). What matters is that the energetics of the material are such that photons cans "see" some subset of electrons - can resonate with them, in particular - but for some more complicated set of energetic reasons related to the mirror material, the photons cannot easily be absorbed by those same electrons. (An image: Picture of millions of delicious healthy snacks behind a glass wall. You can see all of them, all at once, yet you cannot get hold of any one of them.)

In that situation, all of those absorptions become potentials only, not actualities. Thus once again, the Feynman path integrals of "things that could be but were not" kicks in and creates the interference pattern we know as reflection.

The point is that Gary is exactly right, and we are exactly wrong, because reflection is the opposite of absorption. Absorption is what happens when a photon is taken in by an electron and dismantled by it. Reflection is what happens when a photon could be taken in by an electron, but due to energy barriers or quantum chance, it is not.

A reflection - a phased sum of things that did not happen - can occur from a single electron (Compton scattering), a single atom (coherent Compton scattering), or a giant solar sail. The size, delightfully, makes no difference at all because reflection is the sum of "what did not happen", and the universe of what did not happen can be as large as you want it to be. In the case of Gary's other interest, the cosmic microwave background, that scope of things that did not happen can include, literally, the entire visible universe.

Now, this is where things get interesting.

Look back at the two Compton scattering examples I mentioned, free electron and atoms. I just asserted that both standard free-electron Compton scattering and atomic (coherent) Compton scattering are examples of reflection and that they differ from reflection by largescale mirrors only in scale. That's because for both of these cases - even the free-electron case, somewhat surprisingly - the photon is altered but not dismantled. Thus the photon behavior after such interactions is defined by Feynman sums of what did not happen, that is, what is undetectable classically.

Eh? Uh, Terry, dude, you may have lost the bubble here, big time. Since when is an electron or atom sailing off at high speed after a Compton photon encounter "undetectable"? Also, have you seen the Hubble Pillars of Creation images in which photons from off-camera hot stars have cleared vast regions of space to create incredibly photogenic columns of dust? What about those nicely precise Lebedew and Nichols radiometer results from a century ago that proved that mirror reflection is the best way to use photons to push classical objects around? Finally, what about solar sails?

So how can you claim any of these examples of reflection is "undetectable" classically when all of them deposit momentum that is trivially and classically detectable?

It's not complicated: Feynman got it wrong. His paths of possible histories are not histories at all. They are the real paths in xyz space of previously unrecognized particles - vacuum phonons [1] - that carry very small quantities of pure linear momentum. Feynman's paths resemble histories only because vacuum phonons are the particles responsible for defining the locations of objects in xyz space. In solid matter, ordinary phonons - quanta of sound - are dressed-up quasiparticle versions of these same more fundamental vacuum phonons.

Since every object has a Feynman path integral, every such object also radiates a net-zero-momentum (from its own frame) field of vacuum phonons. The more you try to pin an object down in xyz space, the more energetic and its vacuum phonon field becomes. But the particles are quite real and are just as limited by the speed of light as any other particle.

Given all that, a better way to represent what is going on is to abandon path integrals and replace them with a fibrous, xyz-embedded (not Hilbert space) version of Schrodinger wave packets. Inside the wave packet, you have Feynman paths, reinterpreted as the mutually interfering paths of vacuum phonons. At the outer edge of the linear momentum wave packet you have what might best be described as an object-location bubble - and the 
bigger the bubble gets, the less stable the object location becomes. This part I'm still exploring, and it's fascinating. The bound mass of the objects encountered by the ...

[naming tangent: vacuum phonon is too cumbersome. how about vacuum phonon $\rightarrow$ "vonon"? short, easy to say, clean on a Google search, but it also has non-trivial visual and auditory resonances to "venom", so no. space phonon $\rightarrow$ "sphonon"? nice, but "sf" is an uncommon consonant blend for most English speakers. space phonon $\rightarrow$ "sonon"? clean on Google, easy to pronounce, and has very apt resonances with sound waves via sonar and sonic. conclusion: sonon]

... sonon field interacts with the sonon field at a quantum level via on-off mass shell effects. Massive target objects gain all the momentum that hits them but very little energy. Objects with tiny masses, especially ones that resonate well with the entity radiating the field, in contrast, are capable of capturing a sizable chunk of the available energy of the field.

Dr. Kastner, somewhere in this scenario of "delicate oversized bubbles" is the instability leading to symmetry breakdowns - that is, to the true, physically meaningful wave collapses you are seeking. You don't get infinitelength, block-universe-style OWs (retarded waves) and CWs (advanced waves) as Wheeler and Feynman assumed, but you do get a beautifully localized version of the same situation over the much smaller (and again, quite real) 4-space of the sonon field. (I've not even gotten into the delicious time aspects of all of this, oh my!)

The block universe is unnecessary in any case. Einstein was pre-computer and just kind of punted on that one. All his SR really says is "you only need one canonical causality frame, but you can never, ever tell by any local test whether or not you are in that frame." (The more precise statement, one both Hume and pre-Minkowskiconversion Einstein would have loved, is that each observer creates their own space and time, with none more fundamental than any other. It's only when you stitch these isolated realities together - using sonon fields, incidentally - that realities with less total mass-energy end up subjugated to slower comparative clock times.

You, dear reader, are part of the causal frame fabric right now since reading is inherently causal. But more importantly for this argument, having only one canonical frame with entirely real causal time flow is exceedingly helpful for reconciling the Lagrangian views of WheelerFeynman and Kastner block universes with the single-time perspectives of most Hamiltonian representations.

The resolution is this: Classical time flow is real, just "jagged." It's full of quantum regions that remain unresolved in both the past and future when mapped into classical time, and it is within those spindle-shaped regions of quantum uncertainty that the Feynman path integral approach, modified to use sonon fields (QED without momentum transfer by sonons is just wrong) comes into full effect.

(Somewhere way back in my paper notes [I'll look for it] is the Langolier function. With a voraciousness that increases exponentially over time, this function consumes lingering images of the undefined past through the process of quantum collapse. Steven King is a more astute quantum cosmologist than folks realize.)

In the case of Dr. Kastner's symmetry breaks that "choose" between various CW returns, a Langolierian spacetime provides a straightforward solution: The breaks are determined by the distribution of mass and energy of each double-cone, spindle-shaped "sonon bubble" in the fabric. The edge distributions are going to be the most critical to this process since strong absorbers with very low masses are more - that's more, not less - likely to "burst the bubble" by causing the mass-energy of the entity attached to the sonon field to teleport (can't think of a better word yet) to that location.

And oh my, the implications that Langolierian spacetime has for fixing up Hilbert-biased General Relativity are delightful and delicious. It's about time we get a theory of large-scale spacetime that actually matches features of the incredibly fibrous, void-filled, and bizarrely asymmetric large-scale cosmos full of inexplicably complex galaxy forms. GR has been a great first approximation, but it begins to fail badly when those momentum fields get too stretched out. MOND is a good first guess due to its recognition of stretching effects, but details of the interacting sonon fields are more complex than that.

[1] T. Bollinger, Proposal of a new spin-0 puremomentum 'vacuum phonon' particle. Backreaction, July 28, 2020, 6:42 PM ET. https://backreaction.blogspot.com/2020/07/einsteins-

greatest-legacy-

thought.html?showComment $=1595976127097 \# \mathrm{c} 336830$ 1160711284521 